\title{
Criminologie
}

\section{Politiques pénales et peur du crime : "Ordre et sécurité » (Canada) et « Sécurité et liberté » (France).}

\section{André Normandeau}

Volume 16, numéro 1, 1983

La peur du crime

URI : https://id.erudit.org/iderudit/017171ar

DOI : https://doi.org/10.7202/017171ar

Aller au sommaire du numéro

Éditeur(s)

Les Presses de l'Université de Montréal

ISSN

0316-0041 (imprimé)

1492-1367 (numérique)

Découvrir la revue

Citer cet article

Normandeau, A. (1983). Politiques pénales et peur du crime : « Ordre et sécurité » (Canada) et « Sécurité et liberté » (France). Criminologie, 16(1), 51-68. https://doi.org/10.7202/017171ar d'utilisation que vous pouvez consulter en ligne. 
POLITIQUES PÉNALES ET PEUR DU CRIME

"Ordre et sécurité " (Canada) et

"Sécurité et liberté " (France).

André Normandeau*

La justice est, par définition, affaire politique.

Casamayor

\section{INTRODUCTION}

Le gouvernement canadien a présenté en 1976 et, en reprise partielle en 1977, deux importants projets de loi ayant trait au droit pénal, sous l'étiquette commune de : "protection contre la violence criminelle : ordre et sécurité publics ". La "loi de 1976 modifiant le droit pénal, $\mathrm{n}^{\circ} 1$ " traite d'une " loi modifiant le Code Criminel (meurtres et certaines autres infractions graves)" alors que la "loi de 1976 modifiant le droit pénal, $\mathrm{n}^{\circ} 2$ ", traite d'une "loi pour mieux protéger la société canadienne contre les auteurs de crimes violents et autres délits ". Ces deux projets de loi ont été acceptés et votés par le Parlement du Canada d'abord sous l'aspect plus spectaculaire de l'abolition totale de la peine de mort en 1976 et ensuite sous l'angle d'une kyrielle d'autres changements en 1977. Ces mesures étaient les suivantes :

1. affectation de ressources accrues à la prévention de la criminalité;

2. nouvelles dispositions relatives à la condamnation des personnes trouvées coupables de meurtre ;

3. programme de réglementation plus stricte des armes à feu, y compris des peines accrues pour les personnes qui utilisent des armes à feu au cours d'une activité criminelle ;

4. dispositions spéciales pour assurer le contrôle des délinquants dangereux ;

5. attaque concertée menée contre le crime organisé en facilitant les enquêtes policières par l'écoute électronique;

6. imposition de procédures de sélection plus efficaces pour la mise en liberté des criminels violents et exercice d'une meilleure surveillance de ces délinquants après leur libération.

Le programme comprenait également des plans destinés à :

- améliorer la formation des agents de police et du personnel des prisons ;

* Professeur titulaire, École de criminologie, Université de Montréal. 
- accélérer la construction de nouvelles prisons plus petites appelées à remplacer les établissements du genre "forteresse";

- aider la police par l'établissement de meilleures mesures préventives ;

- mesurer les conséquences de la criminalité violente pour les victimes et mieux soutenir celles-ci ;

- mieux connaître les réactions du public envers l'administration de la justice.

Les mesures les plus discutées touchaient les nouvelles peines ainsi que les nouvelles restrictions à la libération conditionnelle. Ainsi, si la peine de mort était abolie, les personnes reconnues coupables de meurtre au $1^{\text {er }}$ degré (meurtre planifié et délibéré, meurtre d'un agent de police ou d'un employé de prison, meurtre accompagnant un viol...), purgeront dorénavant une peine de 25 ans d'emprisonnement avant d'être admissibles pour la première fois à une possible libération conditionnelle. Ce minimum est de 10 ans pour un meurtre au $2 e$ degré. La loi pourvoit à la révision par trois juges d'une Cour supérieure, de la date d'admissibilité dès que le détenu a purgé 15 années d'emprisonnement. De plus, d'autres délinquants reconnus coupables de certains types de crimes accompagnés de violence et qui ont en outre un passé violent, ne deviendront admissibles à la libération conditionnelle qu'après avoir purgé au moins la moitié de leur peine (auparavant, la norme était au tiers). La nouvelle législation créait également une catégorie de " délinquants dangereux " passibles de peines de durée indéterminée, c'est-à-dire de peines permanentes sujettes à révision périodique par la Commission nationale des libérations conditionnelles. Le tribunal peut imposer une telle peine si le délinquant a été trouvé coupable d'un délit criminel pour lequel il peut être condamné à dix ans et plus d'emprisonnement et qui a comporté l'emploi ou une tentative d'emploi de la violence ou une conduite dangereuse ou susceptible d'être dangereuse pour la vie d'une autre personne ou susceptible d'infliger de graves dommages psychologiques à une autre personne.

Le gouvernement français, pour sa part, a présenté en 1980 et 1981 un retentissant "projet de loi renforçant la sécurité et protégeant la liberté des personnes". Ce "Projet Peyrefitte ", du nom du ministre de la Justice, a été sanctionné en février 1981. Les points les plus significatifs étaient les suivants :

1. La récidive: Assimilation de nombreuses infractions dites violentes du point de vue de la récidive : menaces / vol avec violence / coups et blessures aggravés / destruction, dégradation, détérioration d'un bien aggravées. Conséquence : doublement des peines ; 
2. Les circonstances atténuantes: Pour les infractions dites violentes, les juges pourront diminuer à trois ans si la peine encourue est égale ou supérieure à vingt ans, à deux ans pour toute peine de dix à moins de vingt ans. En cas de récidive, si la peine encourue est inférieure à dix ans et d'au moins cinq ans, le tribunal peut descendre à un an;

3. La réitération d'infraction : Le doublement du maximum de la peine encourue est entraîné lorsqu'il apparaît dans une même procédure que, dans le délai d'un an, l'intéressé a commis trois des infractions visées au chapitre des circonstances atténuantes;

4. Les sursis : Le sursis simple ne peut plus être accordé pour les infractions dites violentes : 1 . si, cinq ans avant les faits, l'auteur a été condamné pour crime ou délit avec ou sans sursis; 2 . si la peine prononcée est supérieure à deux ans. Il en est ainsi pour le sursis avec mise à l'épreuve lorsque l'auteur a été antérieurement condamné pour crime ou pour un délit dit violent ;

5. Les nouvelles incriminations: Introduit la notion de menace et tentative de menace contre les biens et ne définit plus la menace. Sanctionne "l'intrusion", notion qui n'existait pas. Sanctionne la destruction, dégradation et détérioration d'un bien quelconque.

6. L'exécution des peines : Élargit le champ d'application en multipliant le nombre d'infractions justifiant une période de "sûreté " qui fait que certains condamnés ne peuvent bénéficier pendant ụn certain temps d'adoucissement de l'exécution de la peine (semiliberté, permissions de sortie, libération conditionnelle) ;

7. Les droits des victimes : Permet à la victime de se constituer partie civile par lettre recommandée et prévoit une réduction automatique de la peine à la moitié du maximum légal de la peine encourue en cas d'indemnisation de la victime ; de la même manière, le régime de sûreté et les possibilités de libération conditionnelle en pareil cas sont assouplis.

Nous pourrions ainsi énoncer plusieurs autres mesures qui ont été incorporées en 76-77 au Canada et en 1980-81 en France au droit pénal de ces deux pays à la lumière d'un disccours justificatif relativement similaire des autorités législatives, discours qui se résume sous la rubrique de la peur du crime en Amérique et du sentiment d'insécurité des citoyens en Europe. En fait, nous y découvrirons bientôt le langage de la punitivité. C'est le leitmotiv principal de ce genre de législation. 


\section{DISCOURS DE LA PEUR ET DE L'INSÉCURITÉ}

Nous retrouvons le discours de la peur du crime en parallèle avec la "vision" d'une société de plus en plus violente. Faisant fi des nuances importantes que certains auteurs ont apportées en démontrant à l'aide d'une "histoire de la violence" (Chesnais, 1981) que nous vivons dans une société probablement moins violente qu'autrefois, l'homme public prend pour acquis qu'il y a effectivement plus d'actes de violence et que la population est effectivement plus atterrée. La conclusion qu'il en tire est classique : accroître les peines et le tour est joué !

Le discours canadien est plus sobre que le discours français. Dans les notes accompagnant les projets de loi de 1976, les ministres responsables, Warren Allmand et Ron Basford, écrivent que :

La montée de la criminalité, y compris la criminalité violente, fait comprendre aux Canadiens qu'il faut apporter des modifications au droit pénal et à l'administration de la justice criminelle afin d'assurer la meilleure protection possible au public. L'appareil de la justice criminelle existe avant tout pour protéger la société contre les effets du crime. La criminalité au Canada n'est pas effrénée, mais il est beaucoup plus facile de demeurer maître de la situation que de le redevenir. C'est pour cette raison que le Gouvernement propose d'adopter le programme " ordre et sécurité publics". Les préoccupations du public portent sur les points suivants :

- crimes répétés commis par les accusés en liberté sous cautionnement ;

- augmentation de la criminalité violente;

- difficulté qu'a la police à lutter contre les "intouchables » du crime organisé ;

- quelques crimes spectaculaires commis par des personnes relâchées par les établissements fédéraux, des libérés conditionnels et des détenus en congé temporaire ;

- émeutes et prises d'otages dans les prisons;

- crimes répétés commis par des délinquants qui semblent être des violents chroniques ;

- crimes tragiques et spectaculaires impliquant l'emploi d'armes à feu.

L'État a le devoir de protéger ses citoyens contre les effets du crime, surtout de la criminalité violente... La justice criminelle est essentiellement un mécanisme de contrôle et n'est pas en ellemême responsable de l'incidence de la criminalité dans la société ni de la prévention de cette dernière. Elle concourt à limiter l'incidence de la criminalité par l'effet intimidant de la peine qu'elle réserve au criminel et à prévenir la criminalité par l'action de la 
police dans ce domaine. Ce qui maintient l'ordre dans la société, ce sont le système de valeurs et l'entretien chez chaque citoyen du sentiment de la responsabilité de ses actes et de la responsabilité de son milieu. C'est notre affaire à tous.

Le discours français, par contre, est beaucoup plus direct. Trois ans avant le dépôt de son projet de loi, le future ministre de la Justice Alain Peyrefitte ${ }^{1}$ déposait le rapport du Comité d'études sur la violence (1977) dans lequel il affirmait que :

Longtemps tenue en marge, la violence s'est installée au cœur de la Cité. Pas encore en maîtresse, mais ce temps peut venir. $\mathrm{Si}$ rien n'est fait pour répondre à l'interpellation qu'elle nous adresse, ce temps viendra sans doute... Un sentiment d'insécurité générale est apparu, qui lui-même peut engendrer la violence, dans une société où la règle du droit n'entraîne plus un consensus général, et où certains sont tentés de se faire justice eux-mêmes. Ce sentiment d'insécurité est à l'origine de ce rapport et de la réflexion qui nous a été demandée... Pour suivre les tours et détours de la violence, nous avions besoin d'un fil conducteur. Nous l'avons trouvé dans le sentiment d'insécurité qui s'est réveillé en France dans les dernières années. Le Comité d'études a estimé de sa mission de chercher à apporter des réponses à cette insécurité, au même titre qu'à la violence, à la criminalité et à la délinquance. Il l'a considérée comme un objet distinct d'investigation... (p. 31, 32, 37).

Le Comité d'études affirme ensuite que "à nouveau, notre temps connaît une certaine peur" (p. 41), que la résurgence de cette vieille crainte est un "phénomène cyclique " (p. 43). "Au long de l'histoire, chaque crise de ce type a provoqué des difficultés que les pouvoirs publics ont cherché à surmonter. Le plus souvent, par des moyens de répression ; quelquefois, par des mesures de dissuasion ; jamais encore, par une politique de prévention sociale" (p. 43). Le Comité de souligner que "depuis 1969, l'angoisse collective sourd à nouveau. Elle se répand brutalement "(p. 46). Et de citer certaines manchettes de journaux des années 70 : "Quand la peur s'installe" (Le Point) ; "Notre époque : les banlieues de la peur " (Le Nouvel Observateur); "Crimes : la carte de France de la peur" (Paris-Match); "Vous sentez-vous en sécurité ? (L'Express) ; et "La société de la peur "(Le Nouvel Observateur).

Parmi les recommandations du Comité, une série s'appliquait à la législation. Le Comité constatait tout d'abord que "Notre législa-

1. Monsieur Alain Peyrefitte est également un académicien émérite qui a écrit sur plusieurs sujets qui ne relèvent pas de la justice pénale. Nous ne référons pas à ce Peyrefitte-académicien le long de notre article mais plutôt au Peyrefitte-politicien au sens strict, compte tenu de nos propos sur les politiques pénales et la peur du crime. 
tion pénale est insuffisamment différenciée ; elle souffre d'un manque de cohésion; elle représente un caractère inflationniste" (p. 65). Il proposait ensuite de : "Poursuivre et accentuer l'effort engagé il y a quelques années en vue de la création de peines nouvelles... " (p. 211).

Le suivi le plus manifeste du rapport du Comité fut précisément le dépôt trois ans plus tard, en 1980, du "Projet Peyrefitte " sur la "sécurité et liberté " des Français. Il s'agissait, disait Peyrefitte, d'un "texte de circonstance". À une question d'un journaliste (Le Nouvel Observateur, 19 mai 1980, p. 59) qui lui signalait que "ce projet paraît être un texte de circonstance, un code de la peur que l'évolution de la criminalité ne justifie pas ", il répondait que :

Les circonstances, étymologiquement, c'est ce qui se situe autour de nous ; c'est bien à cause des circonstances qu'il faut préparer des lois nouvelles, sinon on s'en tiendrait aux anciennes. La question est de savoir comment il faut apprécier les circonstances, c'est-à-dire, l'état de la gestion pénale et de l'insécurité dans la France d'aujourd'hui. Or, il faut malheureusement constater une montée réelle de la violence objective ; une aggravation de l'insécurité subjective; une défiance accrue envers la justice pénale ; la multiplication des actes prétendus d'auto-défense, ou en fait d'auto-justice... Il y a là un grave risque de désagrégation sociale. Dans cette vaste pelote qu'est le Code pénal, il fallait bien tirer par un bout. La priorité que nous avons choisie, c'est la lutte contre les infractions de grande violence. Nous avons déterminé un angle d'attaque : la restauration de la peine et l'accélération de la procédure. Ce n'est pas un texte de circonstance au sens péjoratif. C'est un texte qui correspond aux besoins de la société française du temps présent.

Le leitmotiv était donc clair et net : il s'agissait essentiellement d'une "restauration de la peine", d'un discours de la punitivité, discours ancien emballé à la moderne auquel certains applaudiront et que d'autres rejetteront sévèrement.

\section{DISCOURS DE LA PUNITIVITÉ}

Le discours canadien se rattache directement à $l$ idée traditionnelle de la punition et de son effet intimidant. En conclusion des notes accompagnant les projets de loi de 1976, les ministres Allmand et Basford déclarent qu'on doit chercher la solution à la criminalité et à la violence "du côté de peines sévères mais humaines".

De son côté, le ministre français Peyrefitte est beaucoup plus volubile. Il écrit même sur le sujet un livre au titre suggestif : Les chevaux du lac Ladoga : la justice entre feu et glace (1981). Il avait déjà esquissé 
les grandes lignes de sa conception de la peine lors de son passage à 1'Université de Montréal en 1980. (Le Devoir, 4 juin).

Ce ministre-académicien a le sens de la formule bien faite. Dans son livre de 1981, il y défend ses idées et son projet de réforme pénale. Et de neutraliser la critique : "La justice n'est pas faite pour plaire" écrit-il. "Le feu ou la glace ; comme nous aimons, nous autres Français, ce jeu dangereux ! Nous avons en nous l'esprit de contradiction et sommes réfractaires à l'esprit de transaction ". "La justice n'a jamais été si sollicitée; ni si impopulaire. On se tourne vers des recours contre lesquels on ne cesse de protester". "La critique est utile. Mais toutes les critiques ne sont pas justes. A preuve, leur incohérence".

"Pourquoi punir?", lance Peyrefitte (1981). Il est curieux, constate-t-il, que la notion de prévention soit entourée d'une telle faveur ; la notion de répression d'un tel discrédit. Pour sa part, il prend parti : "La société a le droit de punir. Le discours dominant le nie, le peuple l'affirme" (p. 293). Pour Peyrefitte, dans les pays démocratiques, la notion de répression est inhérente au respect des droits de l'homme : elle ne vient qu'après le délit ou le crime, et au terme d'une procédure minutieusement et contradictoirement organisée. La répression est à la prévention ce que le contrôle financier a posteriori est au contrôle a pricri. Elle est un recul de l'arbitraire et un progrès du droit. Refuser à la société le droit de punir, c'est nier la société ellemême. En s'appuyant à sa façon sur Emile Dunkheim, Peyrefitte affirme le droit de punir. Il s'agit de restaurer le lien de légalité et de solidarité, qui a été brisé par l'acte de violence. La cité punit pour demeurer cité. Elle punit pour survivre. "En tout cas, ce qui doit être affirmé, c'est le droit et le devoir de punir parce que nous sommes libres et solidaires" (p. 301). La société doit accepter de punir. Dans son discours de Montréal en 1980, il avait explicitement défendu cette idée :

Une société qui conçoit mal ses peines conçoit mal ses libertés. Pour le dire autrement : une société qui ne croit plus à la signification des peines ne croit plus à la valeur de la liberté. Car la peine exprime, à sa façon, la vitalité du lien social. Le lien social s'affaiblit et se désintègre si ceux qui ne le respectent pas ne sont pas punis... Les sociétés libérales doivent affronter avec plus de lucidité et plus de courage la question du crime et de son châtiment. Quand une société accepte de punir, elle accepte d'être elle-même, elle accepte de croire en soi. Qu'il y ait des peines ne doit pas nous apparaître comme une survivance choquante de siècles révolus ; cela doit nous rappeler au sentiment du sérieux de ces valeurs essentielles que sont la solidarité, le respect des autres, la responsabilité et la liberté (p. 9). 
La boucle est ainsi bouclée. Si la violence (ou sa perception) s'accroît, si la peur du crime et le sentiiment d'insécurité (ou leurs perceptions) augmentent en intensité, il est temps de réaffirmer le droit de la société à punir plus sévèrement les actes de violence. Les législations canadienne et française sur l'ordre, la sécurité et la liberté répondent à cet enchaînement que les critiques étiquetteront d'infernal. Le Syndicat français de la magistrature (1980), par exemple, frappera sans appel le projet Peyrefitte, le qualifiant de "scélérat":

Il est inutile d'épiloguer sur un texte scélérat, qui tend à isoler un peu plus l'individu face à l'État et à la répression. Ce texte vise tous les mouvements de contestation en criminalisant les actions syndicales et en réduisant le rôle essentiel de la justice. Le magistrat est pris en otage par une législation qui limite son appréciation. Il est soumis à une procédure où le parquet prédomine. Il deviendra davantage garant de la sécurité de l'État que des libertés individuelles. On veut transformer l'appareil judiciaire en une machine à condamnation automatique. Toute cette technologie juridique et judiciaire mise en œuvre par le projet "sécurité et liberté " est étrangement celle des pays totalitaires à l'Est comme à l'Ouest. Plus que de la procédure anglo-saxonne, c'est de la législation du Chili, de l'Uruguay ou de la Tchécoslovaquie que s'inspire le gouvernement. Cela n'a rien d'étonnant alors qu'à l'instar de Pinochet ou de Videla, Monsieur Peyrefitte qualifie déjà de "communistes" ceux qui défendent les libertés de l'individu contre l'envahissement de l'État et de la rationalité économique (p. 1).

\section{RÉACTIONS CRITIQUES}

Les réactions critiques à ces législations ont été très différentes au Canada et en France. Plutôt " douces" (avec nuances !) au Canada. Plutôt "dures" (sans nuances) en France.

Au Canada, l'opposition principale n'est pas venue des partis politiques mais de certains groupes professionnels. Et encore, il ne s'agissait nuflement d'une opposition globale. En effet, les principaux groupes professionnels en la matière étaient tellement enchantés par l'abolition (enfin !) de la peine de mort, que le " troc " leur apparaissait "acceptable". Le gouvernement abolissait la peine de mort mais " serrait la vis " partout ailleurs. C'était, semble-t-il, le prix à payer pour l'abolition! Certains groupes, toutefois, résistèrent à ce jeu. La Société canadienne de criminologie (McGrath, 1976) et la Société de criminologie du Québec (Normandeau, Rico, 1976) s'opposèrent avec vigueur à la sentence d'emprisonnement minimale de 25 ans, à l'introduction 
d'une catégorie spéciale de " délinquants dangereux " et aux nouvelles restrictions apportées à la libération conditionnelle.

Landreville et Petrunik (1981) ainsi que Rico (1981) ont soulevé à juste titre l'hypothèse de la "fonction idéologique " des législations sur les délinquants dangereux. Comme ils le soulignent, malgré les nombreuses remises en question du rôle des experts, de leur capacité de prédire avec succès le comportement violent et les infractions sexuelles graves, ainsi que l'inefficacité des mesures de détention indéterminée, pour les délinquants dits dangereux, la notion de dangerosité est devenue une "notion passe-partout " dans le domaine du droit pénal et de la santé mentale. On adopte une "ligne dure "plus sévère, pour la délinquance grave, celle impliquant la violence ou la menace de violence, et une " ligne douce " pour la délinquance mineure contre les biens où il n'y a pas de violence contre la personne. On apaise ainsi le public. On donne l'impression que le problème est réglé. C'est ce que les auteurs appellent la "fonction symbolique" de telles législations. Cette fonction, d'ailleurs, s'applique à l'ensemble du contenu des législations spéciales sur l'ordre, la sécurité et la liberté des... Québécois, des Canadiens, des Français et autres citoyens. Mais, pourquoi les concepts de violence, dangerosité, peur et insécurité sont-ils toujours aussi importants dans les législations et les politiques? En paraphrasant Landreville et Petrunik (1981), il faut considérer, en premier lieu, la nature des événements violents et la façon dont on les perçoit. Il est probable que l'origine et la persistance des mesures spéciales'de contrôle puissent être en partie expliquées par ce que Merton et Nisbet nomment "la perception sociale des problèmes sociaux" (1982). Une dimension de cette perception sociale est la signification perceptuelle de la distance géographique, sociale et psychologique. Ainsi, la mort de milliers de personnes lors d'un désastre naturel ou d'une révolution dans un pays éloigné, a moins de chance de nous émouvoir qu'un seul meurtre d'un enfant dans notre communauté. Un seul viol ou un seul crime de violence nous préoccupera plus que quelques centaines ou quelques milliers de morts accidentelles sur les routes ou dans les accidents de travail, morts que nous percevons comme "normales" ou " routinières". Les crimes de violence soulèvent l'indignation morale, facilitant la tâche des "entrepreneurs moraux" (Becker, 1963) dans leur campagne pour faire adopter de nouvelles législations, comme l'a démontré Sutherland (1950).

Une autre dimension de cette perception sociale est liée à la perception des causes de l'événement problématique et de ce qui est responsable de son apparition et de son contrôle. Ces perceptions influen- 
ceront même le fait que l'événement sera ou non considéré comme problématique. Les actes de violence sont ainsi considérés comme des gestes d'individus dangereux, individus que l'on doit identifier et contre lesquels on doit se protéger en les internant et même en les supprimant. La plupart des gens suggèrent de tout mettre en œuvre pour neutraliser ces individus dangereux. Ils se préoccuperont moins du fait que cette approche peut conduire à incarcérer de nombreux individus qui, même s'ils ont déjà commis un acte de violence, ne récidiveront pas et ne seront pas dangereux.

Landreville et Petrunik soulignent (p. 219) que les membres du gouvernement sont nécessairement sensibles aux préoccupations ou du moins aux pressions des électeurs. Aussi, même lorsqu'un "problème " ne peut être résolu adéquatement, il est important que le gouvernement "s'intéresse à la question ", propose des solutions et laisse du moins croire qu'il les met en application. Aussi, ces solutions peuvent être "symboliques " plutôt qu'" instrumentales " (Gusfield, 1967). Souvent il importe peu que la nature et l'étendue du problème soient mal évaluées et que les solutions masquent ou déplacent le problème pourvu que le public soit apaisé. Il faut cependant préciser que parfois ceux qui proposent ces solutions veulent croire et arrivent à croire à ce qui est en réalité une pseudo-solution. Une loi peut remplir certaines fonctions même si elle est, d'un certain point de vue, inefficace et peu utilisée.

Enfin, ce genre de loi sert aussi de "diversion". On attire l'attention sur le danger et la violence individuelle plutôt que collective ou structurelle. Les situations ou les conditions de travail mettant en danger la santé des travailleurs, l'ivresse au volant ou la pollution industrielle ne sont pas définies comme dangereuses au point où il faille adopter des mesures spéciales très sévères pour protéger le public. On détourne l'attention d'autres situations ou pratiques dangereuses qui sont généralement celles des classes sociales favorisées. On détourne également l'attention de certains autres problèmes sociaux et politiques. On ignore les questions d'injustice, d'inégalité, les situations répressives qui ont pu engendrer la frustration et l'agression. On pourrait, par exemple, se demander jusqu'à quel point tout le programme canadien "ordre et sécurité publics" mis de l'avant " pour protéger le peuple canadien contre la violence " n'a pas contribué à détourner l'attention de situations très embarrassantes pour le gouvernement canadien comme l'accroissement du chômage ou l'inflation. Cet effet de diversion peut s'exercer qu'il ait été ou non voulu et planifié. 
Il est permis de s'interroger sur les "fonctions politiques" de la notion de "violence " et de "peur du crime" auxquelles la criminologie a été trop peu attentive.

Nonobstant de telles préoccupations "symboliques ", les projets de loi canadiens autour de la sécurité furent votés en 1976 et 1977 sans débat public majeur. En France, au contraire, le débat public de 1980 et 1981 fut d'une rare intensité. Le projet "sécurité et liberté " suscita une véritable mobilisation des principales organisations politiques, syndicales et professionnelles. Le parti socialiste français, alors dans l'opposition, sortit ses "gros canons" contre le projet et son leader François Mitterand promit officiellement de retirer cette loi s'il était élu Président de la République quelques mois plus tard. Au sein même du groupe parlementaire majoritaire, le leader Jacques Chirac, exPremier Ministre et Maire de Paris rejeta le projet globalement.

Le Syndicat de la magistrature, on l'a vu plus haut, fut le plus dur. Selon le Syndicat, l'individualisation de la peine, la réinsertion sociale étaient remplacées par l'élimination systématique de toute une catégorie de la population pénale, originaire des classes les plus défavorisées. Il s'agissait d'une " réforme en trompe-l'œil ", dissimulant une généralisation d'une justice expéditive et orientée vers une répression qui ignore totalement la délinquance économique, au coût social le plus élevé. "Ce texte, écrivait le Syndicat, n'est que l'aboutissement du discours démagogique sur la sécurité... Le nombre de détenus va doubler dans les années à venir. L'augmentation du nombre de petits délinquants qui auront à subir l'influence criminogène de la prison n'est pas une garantie pour la liberté et la sécurité des Français" (1980, p. 2).

Le critique de l'opposition socialiste, Robert Badinter, qui devait devenir le prochain ministre de la Justice sous un gouvernement Mitterand-Maurois quelques mois après, décrivit le projet de loi comme un " tour de passe-passe", une " mystification ", un "gadget", un " texte-arlequin ", un " chef-d'œuvre d'humour noir " et un " projet monstrueux".

Même des juristes-criminologues associés à certains travaux du gouvernement, comme MM. Jacques Léauté et Georges Levasseur, malmenèrent le projet. "Il ne suffit pas d'amender ce texte, il faut le rejeter complètement ", déclara Léauté. Et Levasseur d'affirmer que "si le projet a obtenu un incontestable succès de surprise, les protestations contre son contenu ont fusé, à juste titre... et les justifications avancées pour le présenter ou le défendre n'ont convaincu que bien 
peu de spécialistes de la matière. Un vieux routier du droit répressif et pénitentiaire ne peut qu'exprimer sa désapprobation et son inquiétude devant la plupart des dispositions prévues " (voir le dossier de presse du Centre de recherche et de formation de l'éducation surveillée, 1980).

Les critiques professionnelles les plus explicites concernaient la " répression" du crime par l'établissement d'une catégorie spéciale d'infractions "de violence" et par l'aggravation des peines, souvent portées au double.

D'autres arguments percutants furent déposés sur la place publique : texte élaboré ciandestinement, par une poignée d'amateurs dévoués au ministre, dans le dos de la direction des affaires criminelles, institutionnellement chargée de préparer la loi ; rejet des travaux de la commission de révision du Code pénal instituée en 1974 ; manque scandaleux de concertation avec les syndicats de magistrats et d'avocats ; viol de la constitution et des principes généraux du droit ; aggravation des pouvoirs de l'exécutif, au détriment des juges et au mépris de l'indépendance de la magistrature; atteinte grave aux droits de la défense ; rupture avec une révolution séculaire vers plus d'humanité envers les inculpés et les détenus ; régression vers le Moyen-Âge...

Le ministre fut même attaqué directement sur le plan personnel. Le magazine Justice du Syndicat de la magistrature le traitant d'homme "aussi maladroit qu'il se croit habile". Et d'ajouter : "Un jour, certainement, ses déclarations feront l'objet d'un sottisier pour la bonne joie des générations futures " $(1980$, p. 2$)$. Peut-on imaginer pareille écriture dans un journal de juges québécois ou canadiens? Mais la France, c'est la France. Le débat est "viril " (sans sexisme !), même s'il est quelquefois "en bas de la ceinture".

La philosophie pénale du ministre fut évidemment contestée. Même au Québec, où son discours de Montréal sur la peine et le châtiment fut qualifié " d'utopie punitive " par le criminologue et philosophe québécois Jean-Paul Brodeur (1980) :

Je pense, comme M. Peyrefitte, qu'il existe des comportements qui portent préjudice à autrui ; j'estime en outre, comme lui, qu'ils doivent être dénoncés et que des mesures doivent être prises pour en réduire la fréquence. Je ne crois cependant pas que l'escalade des sanctions pénales constitue le recours exclusif pour parvenir à ces fins; je suis en outre convaincu, me fondant sur des descriptions mille fois reprises des dysfonctionnements de la justice pénale, que l'accroissement de la rigueur des peines contrevient à la réalisation des fins précitées... En réactivant une croyance désuète dans les vertus du châtiment, considérée 
comme une panacée dont la prescription devrait être généralisée, M. Peyrefitte nous reporte plus de quarante années en arrière (p. 8).

En retour, même si une majorité des intervenants qui s'expriment publiquement étaient opposés profondément au projet, quelques juristes, en particulier, l'appuyèrent. Peyrefitte lui-même, dans son livre (1981), p. 146), cite des manifestes favorables. Un article plus technique de Rassat (1981) a défendu le bien-fondé criminologique d'un régime particulier pour les infractions dites de violence. De plus, l'auteur réfute l'accusation de "répressif " accolée au projet. L'argumentation à ce sujet serait objectivement fausse car le texte de loi, s'il refond un grand nombre d'infractions anciennes, n'en crée aucune nouvelle : " Le seul argument qui pourrait être soutenu avec quelques vraisemblance consisterait donc à dire que notre droit pénal étant un droit de répression sociale, il le demeure, mais certainement pas qu'il le devient" (p. 12).

Le projet de loi continua néanmoins son chemin et fut sanctionné le 2 février 1981, sans changement notable. "La justice n'est pas faite pour plaire ", disait Peyrefitte. En pilotant son dossier, Peyrefitte fit son "chemin de croix " en toute "lucidité ". Il rappelle dans son livre "l'immense charivari que fit naitre son projet. Il fallut passer par tous les stades de cette sorte de paludisme dont souffre la vie française et qui fait alterner des accès réguliers de fièvre intense avec de longues périodes paisibles. Avant le vote à l'Assemblée, les clameurs d'une minorité : elles couvriront le silence d'une majorité au point même de la troubler. Après le vote, le silence de la minorité, qui laissait enfin percer la satisfaction de la majorité - majorité des Français, majorité des parlementaires, majorité des magistrats" (p. 144).

Un homme politique et un ministre tient évidemment à cet appui de la majorité. Faute, souvent, de mesures objectives à ce sujet, il l'imagine, il l'invente, il la suscite subjectivement. La conception de l'opinion publique de Peyrefitte est semblable à celle de la plupart des politiciens. Les images qu'il utilise sont significatives. Au sujet du projet " sécurité et liberté ", il constate qu'il a essuyé "la charge de la brigade légère... Et pourtant, assez puissante pour faire illusion sur sa force. Le jour même du Conseil des ministres qui adoptait le projet, un cavalier, lance au poing, commençait à le déchiqueter". Il lançait son "J'accuse ", en émule de Zola. Agitant les mêmes armes, "les escadrons suivirent et l' artillerie des partis d'opposition et de presque tous les syndicats donna à tout hasard ; comme ils le font par habitude... chaque fois qu'ils peuvent craindre de le voir satisfaire le public à leurs 
dépens. Nous eûmes beau réfuter les arguments l'un après l'autre, ce fut comme si nous n'avions rien dit... Ces arguments ne pénétrèrent pas plus dans la presse et dans l'opinion, que l'eau sur les plumes d'un cygne. Ce fut un véritable phénomène d'intoxication collective. Chez beaucoup de citoyens de bonne foi, la perplexité finit par naître (p. 145).

Mais le ministre s'y attendait, dit-il, et il était prêt à la bataille en s'appuyant sur l'opinion publique : "Les divers groupes de pression ne s'entendent entre eux que pour réclamer des changements abstraits et bloquer les changements concrets. La seule méthode pratique pour crever l'écran du microcosme judiciaire est d'atteindre le grand public, pour le rendre arbitre du débat. Après tout, n'est-ce pas naturel, quand il s'agit de la loi pénale, qui touche la société en son cœur? N'est-ce pas même la seule méthode admissible ? À moins de professer, selon le mot de Valéry, que la politique a été pendant longtemps l'art d'empêcher les gens de se mêler de ce qui les regarde avant de devenir l'art de consulter les gens sur ce qu'ils ignorent" (p.147).

Et Peyrefitte d'asséner le "coup de grâce " à ses opposants par une métaphore "choc" sur les "pythies désavouées":

Parce que ceux qui prennent la parole ou la plume prétendent parler au nom du peuple souverain, ils occultent la volonté de l'ensemble des citoyens. Ces pythies, enivrées de leurs propres fantasmes, $y$ lisent le message du dieu. Cela fait impression tant que le dieu laisse, sans méfiance, faire et dire. Mais il arrive qu'il se réveille et ne se reconnaisse pas dans l'interprétation des pythies. Cette fois, les clameurs étaient si fortes qu'elles ont réveillé le dieu. Réveillé, il a parlé. Si nos adversaires n'avaient pas ameuté le public, ils auraient pu mettre le projet en panne, dans des discussions de techniciens. Mais ils voulurent soulever bruyamment l'opinion; alertée par leurs soins, elle s'exprima. $\mathrm{Ce}$ fut pour les désavouer.

La majorité, dans notre pays, s'exprime sans fracas. Certains prennent cette discrétion pour du silence. C'est qu'ils se bouchent les oreilles, ou ne savent pas écouter ; à moins qu'ils soient coupés du peuple. Il n'est pas besoin de manifester pour s'exprimer, de crier des slogans pour faire connaître sa conviction... Les parlementaires qui lisent attentivement leur courrier et prennent le temps de faire parler leurs visiteurs dans leurs permanences, la connaissent, eux, cette majorité ; elle dépasse largement leur propre électorat ; ils voient bien qu'elle n'est pas silencieuse. Les élus du peuple savent ce que veut le peuple. Les opposants eux-mêmes, à mesure que se développait leur campagne, remarquèrent que le peuple n'était pas de leur côté (p. 151). 
Peyrefitte avait peut-être oublié que les "élus du peuple " ne sont pas seulement du côté du parti au pouvoir. Il y a aussi les élus de l'opposition, cette opposition dite de gauche en France qui devait quelques mois plus tard prendre le pouvoir.

Que ferait le nouveau gouvernement socialiste de cette loi qu'il avait dénoncée avec véhémence? Il avait promis de la retirer "au complet ». Mais le pouvoir est le pouvoir. Le Gouvernement Mitterand-Maurois a d'abord créé une commission d'enquête (une autre!) pour revoir la situation... et plus d'un an après son arrivée au pouvoir, la loi "sécurité et liberté " est toujours la loi du pays.

Le changement en matière de justice est difficile et excessivement lent si on regarde par comparaison les changements socioéconomiques. Est-ce parce que la justice est "le dernier wagon du train "et n'est pas habituellement une priorité gouvernementale ? Ou est-ce " la leçon de Portalis " qui porte fruit ? Portalis, ce juriste français du XIX ${ }^{e}$ siècle, qui affirmait qu'il faut être "sobre de nouveautés en matière de législation parce que, s'il est possible, dans une institution nouvelle de calculer les avantages que la théorie nous offre, il ne l'est pas de connaître tous les inconvénients que la pratique seule peut découvrir, et qu'il serait absurde de se livrer à des idées absolues de perfection, dans des choses qui ne sont susceptibles que d'une bonté relative. Les lois doivent ménager les habitudes, quand ces habitudes ne sont pas des vices" (cité par Peyrefitte, 1981, p. 107).

Et l'ironie du sort pour le gouvernement socialiste serait qu'il applique, inconsciemment peut-être, cette maxime de Peyrefitte luimême qui disait qu'il faut "continuer avant de commencer " et que "la première réforme, c'est d'appliquer celles qui ont été décidées" (p. $103)$.

Il en est souvent ainsi de nos propres gouvernements en Amérique, au Canada comme au Québec, où, quelle que soit la couleur politique du temps, les réformes en matière de justice sont fort parcimonieuses?

\section{CONCLUSION}

Dans un texte sur "l'évaluation des politiques criminelles", Szabo (1981) souligne qu'une activité de réforme systématique, similaire à ce qui existait dans le domaine de l'éducation et de la santé publique, se fait jour en ce qui touche l'administration de la justice. Que, très rapidement, le problème de l'évaluation des nouvelles politiques de 
défense sociale mises en œuvre est devenu une préoccupation des pouvoirs publics et a commencé à retenir l'attention des milieux universitaires. La politique criminelle serait entrée ainsi dans le champ distingué des "policy sciences" (Szabo, 1978).

Toutefois, le champ est très peu développé. Il y a d'abord des ambiguités, voire des contradictions à relever au niveau des objectifs du droit pénal et des services responsables de son application (Brodeur et Landreville, 1979). De plus, en ce qui concerne le choix et la construction des indicateurs de mesure, notre domaine est moins bien pourvu que les autres secteurs de politique sociale. Il n'est donc pas très surprenant que, malgré les bonnes intentions, aucune évaluation serrée de la loi canadienne de 1976 sur la sécurité n'ait été réalisée (sauf une évaluation préliminaire du chapitre de la loj sur le contrôle des armes à feu). Il est probable aussi que les politiciens et les administrateurs ne soient pas intéressés à une démarche évaluative jugée "menaçante" (Hasenpusch et Normandeau, 1980).

Pour l'instant, l'analyse de telles législations se limite aux premières phases du processus politique qui est souvent découpé en six phases successives, à savoir : 1) la formation de la politique, 2) sa formulation, 3) sa mise au point, 4) sa mise en œuvre, 5) son évaluation et, l'enchaînement étant circulaire, 6) sa reformulation, laquelle devient, de nouveau, un facteur dans la formation des politiques.

Les débats que nous avons résumés au sujet des lois canadiennes et françaises sur la sécurité relèvent de la formation et de la formulation des politiques pénales. Nous y constatons que la sciences des politiques se heurte au problème majeur dans la formation des politiques, celui de l'ambiguïté volontaire (pour ne pas dire obligatoire) des législations promulgées. En effet, celles-ci résultent de nombreux compromis entre des intérêts parfois contradictoires, mais qu'on doit ménager. Leur chance d'être adoptées, du moins en démocratie de type occidental, dépend fréquemment de l'institutionalisation cette ambiguïté, sinon des contradictions, dans la législation même. C'est à cette seule condition que les coalitions d'intérêts complexes peuvent appuyer une mesure législative concrète : plus l'appui est large, moins les objectifs doivent être clairs, moins la philosophie doit être explicite. Pour sa part, la formulation des politiques exige, de la part des administrateurs intéressés, la prise en compte des contraintes politiques, fiscales et bureaucratiques.

En fin de compte, toutes les analyses sont basées sur des analyses intuitives concernant les alternatives à retenir, les résultats à considé- 
rer, et l'appréciation de l'utilité de telle ou telle décision. Dans ce sens, le processus de prise de décision demeure davantage un art qu'une science.

Dans " un " sens, c'est rassurant car la décision demeure au niveau de l'humain, avec ses qualités et ses faiblesses. Dans un "autre" sens, c'est décourageant parce qu'il s'agira d'un recommencement perpétuel.

Y a-t-il une "ligne de progrès " linéaire et continue pour la justice? En "criminologie scientifique", nous y aspirons. Mais est-ce utopique?

Michel Foucault, l'auteur du livre célèbre Surveiller et punir (1975), le voit ainsi quand il déclare (Le Nouvel Observateur, 28 mai 1979) :

Je ne suis guère convaincu quand on me dit que les libertés aujourd'hui sont entamées, que les droits s'effritent et que les espaces se resserrent autour de chacun de nous. La justice pénale d'il y a vingt ans ou d'il y a un siècle, je parie qu'elle n'était ni mieux ordonnée ni plus respectueuse. Inutile, pour dramatiser le présent, d'en allonger les ombres par les clartés imaginaires d'un soleil en déclin.

Les législations " ordre, sécurité et liberté " ne seraient-elles vraiment que les "clartés imaginaires" du soleil séculaire et millénaire de la "peur " de la criminalité violente et de l'" insécurité " des femmes et des hommes de tous les pays, à toutes les époques?

La justice est-elle vraiment entre feu et glace... pendant les siècles des siècles?

\section{BIBLIOGRAPHIE}

ALLMAND, W. et BASFORD, R. (1976) : Protection contre la violence criminelle : ordre et sécurité publics, Loi de 1976 modifiant le droit pénal, $\mathrm{n}^{\circ} 1$ : loi modifiant le Code criminel (meurtres et certaines autres infractions graves); Loi de 1976 modifiant le droit pénal, $n^{\circ} 2$ : loi pour mieux protéger la société canadienne contre les auteurs de crimes violents et autres délits, Ottawa, Gazette officielle du gouvernement du Canada, 1976 et 1977. N.B. : voir également les débats du Parlement canadien sur le sujet en 1976 et 1977 reproduits dans la Gazette officielle.

BECKER, H. (1963) : Outsiders, New York, Free Press.

BRODEUR, J.P. (1980) : "L'utopie punitive ", Montréal, Le Devoir, 10 juin 1980, p. 8.

BRODEUR, J.P. et LANDREVILLE, P. (1979) : Planification des politiques : les finalités du système de justice, Ottawa, Solliciteur général du Canada.

CENTRE DE RECHERCHE ET DE FORMATION DE L'EDUCATION SURVEILLÉE (1980) : "Le projet sécurité et liberté : éléments pour un dossier ", Marginalités, 9, 89.104.

CHESNAIS, J.C. (1981) : Histoire de la violence, Paris, Laffont. 
COLLECTIF DE LA REVUE ACTES (1980) : "Le dossier sécurité et liberté ", Actes, vol. 29 , p. $20-40$.

GUSFIELD, J. (1967) : " Moral passage : The symbolic process in public designation of deviance", Social Problems, vol. 15, p. 175-188.

HASENPUSCH, B. et NORMANDEAU, A. (1980) : " Prevention Programs and their Evaluation ", Canadian Journal of Criminology, vol, 21, p. 307-319.

KEGELS, M.L. (1982) : "Le crime puisqu'il faut l'appeler par son nom... La peur du crime ", Déviance et Société, vol. 6, p. 209-220. (Après la rédaction de cet article, nous avons reçu la revue Déviance et Société où paraît une "Actualités bibliographiques " sur le thème de la peur du crime. Nous y référons le lecteur.)

LANDREVILLE, P. et PETRUNIK, M. (1981) : "Le délinquant dangereux dans les législations nord-américaines ", in C. Debuyst (dir.), Dangerosité et justice pénale, Genève, Médecine et Hygiène, p. 207-230.

LE MATIN DE PARIS (1980) : " La justice en jugement ", numéro spécial hors-série, juin, $36 \mathrm{p}$.

McGRATH, W. (1976) : Ordre et sécurité publics, Mémoire de la Société canadienne de criminologie, Ottawa.

MERTON, R.K. et NISBET R. (1982) : Contemporary Social Problems, 6e éd., New York, Harcourt.

NORMANDEAU, A. (1979) : "Canada - ordre et sécurité publics", in B. Holyst (dir.), Criminologie, Varsovie, Pologne, p. 225-237.

NORMANDEAU, A. et RICO, J. (1976) : Ordre et sécurité publics, Mémoire de la Société de criminologie du Québec, Montréal.

PEYREFITTE, A. (1977) : Réponses à la violence : rapport de Comité d'études sur la violence, la délinquance et la criminalité, Paris, Presses Pocket.

PEYREFITTE, A. (1980) : " Le crime et son châtiment ", Montréal, Le Devoir, 4 juin 1980, p. 9.

PEYREFITTE, A. (1981 a) : Loi n $81-82$ du 2 février 1981 renforçant la sécurité et protégeant la libenté des personnes, Paris, Journal officiel de la République française, 3 février 1981. N.B. : voir également les débats de l'Assemblée nationale sur le sujet en 1980 et 1981 reproduits au Journal officiel.

PEYREFITTE, A. (1981 b) : Les Chevaux du lac Ladoga : la justice entre feu et glace, Paris, Plon.

RASSAT, M.L. (1981) : "Le nouveau régime des infractions pénales dans la loi " sécurité et liberté ", Revue internationale de criminologie et de police technique, vol. 4, p. 7-26.

RICO, J. (1981) : "Les législations hispano-américaines de dangerosité sociale ", in C. Debuyst (dir.), Dangerosité et justice pénale, Genève, Médecine et Hygiène, p. 231-258.

SYNDICAT DE LA MAGISTRATURE (1980) : "Dossier sécurité et liberté ", Justice, vol. 77, p. 1-32.

SZABO, D. (1978) : Criminologie et politique criminelle, Paris et Montréal, Vrin et P.U.M.

SZABO, D. (1981) : "L'évaluation des politiques criminelles ", Revue de science criminelle et de droit pénal comparé, vol. 50, p. 1-23. 